sich selbst machen? Welche Arbeit soll man an sich verrichten? Wie soll man sich mittels Handlungen sregieren (se gouverner)<, bei denen man selbst das Ziel, der Anwendungsbereich, das verwendete Instrument und das handelnde Subjekt ist? « (Foucault [1981] 2016: 377f [Herv. i. O.]) Für Foucault besteht darin die »Verehrung oder der Kult, die man sich selbst gegenüber schuldig ist « (Foucault 1982 in Gros 2009: 660) und zwar die Arbeit an einem Selbst, an dem Geschick, das sich aus dem eigenen Leben machen ließe. Wie bereits in der antiken Praxis des Selbst, ist zu dieser Veränderung ein Lehrmeister notwendig, dessen Rolle auch heutzutage unerlässlich ist (in Form einer Fitnesstrainerin, eines Business Coaches, eines geistlichen Begleiters usw.). Das Subjekt ist, heute wie damals, in der Unwissenheit, Lasterhaftigkeit oder Missbildung befangen und seine Befreiung muss es, laut Foucault, nicht durch ein Wissen anstreben, sondern durch die Erlangung eines Zustands, »den es nie, zu keinem Zeitpunkt seines Daseins, gekannt hat. Es hat den Zustand des Nicht-Subjekts durch den des Subjekts zu ersetzen, der durch die volle Entfaltung der Beziehung zu sich selbst definiert ist.« (Foucault [1982] 2009: $169)^{13}$

Mit dieser Aufforderung gipfelt Foucaults langjähriges Werk, das wegen seinem frühen Tod im Jahr 1984 beenden war. Die hinterlassenen analytischen Mittel und Verfahren inspirieren zu einer radikalen Hinterfragung eingefahrener Denkschemen und bieten eine Möglichkeit an, die Spielräume der Macht aufs Neue zu eröffnen und damit die Vorstellung von der existierenden (Regierungs-)Ordnung zu überdenken. Darauf weist vor allem sein Denken über das Regieren und seine Vorstellung des Politischen hin, die im Konzept der Gouvernementalität enthalten sind.

\title{
3 Gouvernementalität - ein Denken über das Regieren
}

Die beschriebenen analytischen Vorgänge ermöglichen einen Blick in Foucaults Werkzeugkasten. Foucaults Denken und seine Verfahren haben Eingang auch in die politikwissenschaftlichen Untersuchungen gefunden. Vor allem sein Denken über die Regierungstechnologien, die er im Begriff der Gouvernementalität subsummiert hat, inspirierte einen ganzen Forschungsbereich - die GouvernementalitätsStudien -, der sich mit den Fragen des Regierens und Regiert-Werdens befasst.

13 Am Beispiel der Homosexualität offenbart Foucault sein Interesse, Neues zu erzeugen, statt sich in bekannte Modelle einzureihen. Deshalb wollte er nicht wissen: »Wer bin ich? Was ist das Geheimnis meines Begehrens?« (Foucault [1981] 1985: 85), sondern vielmehr: »Was für Beziehungen können über die Homosexualität aufgebaut, entworfen, erweitert und von Fall zu Fall verschieden gestaltet werden?« (ebd.) 


\subsection{Grundzüge des Konzepts}

Das Konzept der Gouvernementalität präsentierte Foucault in einer 1978 gehaltenen Vorlesungsserie, auch unter dem Namen Sicherheit, Territorium, Bevölkerung bekannt (vgl. Foucault [1978] 2006). Während dieser Vorlesungsreihe kulminiert sein Interesse in einer Art spezifischer Betrachtung des Regierungswesens. Der moderne Staat, welcher sich seit dem Mittelalter bis hin zur Gegenwart entwickelt hat, funktioniert seiner Ansicht nach als ein komplexes System von Führungen. So wie die Familie nach gewissen Regeln funktioniert, wo jedem Mitglied eine spezifische Position zukommt, wo jeder mit typischen Aufgaben ausgestattet ist, wo er die Regeln und Strafen kennt, wird auch in anderen gesellschaftlichen Einheiten (wie z.B. Gemeinden, Verbänden, Unternehmen, Kommunen, staatlichen oder nichtstaatlichen Institutionen, Staaten, internationalen Organisationen) eine spezifische Regierungsart betrieben. Von der Familie bis hin zur Regierung der Bevölkerung erstreckt sich demnach eine Vielfalt von Führungen bzw. Regierungen: »Damit der Staat funktioniert, wie er funktioniert, muss es vom Mann zur Frau oder vom Erwachsenen zum Kind sehr spezifische Herrschaftsverhältnisse geben, die ihre eigene Konfiguration und ihre relative Autonomie haben.« (Foucault [1977] 1978b: 110)

In dieser ganzen »Kaskade von Abhängigkeitsverhältnissen« (Wessel 2015: 642) entfalten sich im Übergang von Regierungsweisen des 16. Jahrhunderts durch die ökonomischen Lehren des 17. Jahrhunderts - wie die Theorie des Merkantilismus oder der Physiokraten - spezifische Machtbeziehungen, die auf Selbst-Disziplin beruhen. Die technologischen und industriellen Fortschritte, die damit einhergehende Urbanisierung und die rasche Entwicklung neuer Märkte durch die Entdeckungsreisen und Kolonisation haben die Regierungen des 16. und 17. Jahrhunderts vor neue Aufgaben gestellt und gleichzeitig neue Regierungsgegenstände wie die bürgerliche Bevölkerung - hervorgebracht. Um das Ensemble an Verhältnissen $\mathrm{zu}$ überwachen, tritt eine Macht hervor, die, wie ein Hirte, »ein wachsames Auge auf alles und auf jedes [somnes et singulatim火] « hat (Foucault [1978] 2006: 191f [Herv. i. O.]). Eine Macht, die wie in der Skizze von Benthams Panoptikons (vgl. Foucault [1975] 2008) die Überwachung durch Selbst-Überwachung bzw. Regierung durch Selbst-Regierung erzeugt. Diese Macht reagiert auf spezifische Probleme der Bevölkerung, die zutage treten (vgl. Foucault [1978] 2006: 134) so, dass sie auf jeder Ebene institutionalisierter Verhältnisse - Familie, Schule, Spital, Gefängnis, Armee, Werkstatt - die Entwicklung selbst-disziplinierender Beziehungen fordert, die im Endeffekt auf eine totalisierende, eine globale Institution - den Staat - hinauslaufen (vgl. a.a.O.: 178). Diese Gesamttechnologie der Macht, die die verschiedenen Instanzen durchkreuzt und in die Etablierung und das Festhalten des Staates mündet, nennt Foucault Gouvernementalität (vgl. a.a.O.: 180). Mit diesem Begriff erfasst Foucault den Zusammenhang zwischen den Herrschaftstech- 
niken des Regierens (franz. gouverner) und der Denkweise und den Praktiken des Sich-selbst-Regierens der Subjekte (franz. mentalité), die sich in dem modernen Staat herausgebildet und zur Etablierung neuer Machtverhältnisse geführt haben:

»Ich verstehe unter >Gouvernementalität ‘ die aus den Institutionen, den Vorgängen, Analysen und Reflexionen, den Berechnungen und den Taktiken gebildete Einheit, welche es erlauben, diese recht spezifische, wenn auch sehr komplexe Form der Macht auszuüben, die als Hauptzielscheibe die Bevölkerung, als wichtigste Wissensform die politische Ökonomie und als wesentliches technisches Instrument die Sicherheitsdispositive hat. Zweitens verstehe ich unter >Gouvernementalität`die Tendenz oder die Kraftlinie, die im gesamten Abendland unablässig und seit sehr langer Zeit zur Vorrangstellung dieses Machttypus geführt hat, den man über alle anderen hinaus die >Regierung`nennen kann: Souveränität, Disziplin, und die einerseits die Entwicklung einer ganzen Serie spezifischer Regierungsapparate [und andererseits] die Entwicklung einer ganzen Serie von Wissensarten nach sich gezogen hat. Schließlich denke ich, daß man unter >Gouvernementalität den Vorgang oder vielmehr das Ergebnis des Vorgangs verstehen sollte, durch den der mittelalterliche Staat der Gerichtsbarkeit, der im 15. und 16. Jahrhundert zum Verwaltungsstaat wurde, sich nach und nach >gouvernementalisiert< hat.«(a.a.O.: $162 f$ [Herv. i. O.])

Mit dem Konzept der Gouvernementalität hat Foucault das Problem des Regierens und das Problem des Staates auf neue Weise beleuchtet. Der Staat lässt sich demnach nicht mehr nur durch seine Geschichte, seinem Aufbau, seine Gewaltausübung betrachten, sondern auch als Resultat der »Verstaatlichung einer bestimmten Anzahl von Praktiken, Handlungsweisen [...] einer Gouvernementalität.« (Foucault [1984] 2000: 69) Die »Gouvernementalisierung des Staates« (Foucault, [1978] 2006: 163) zu untersuchen heißt dann, einen Begriff des Regierens anzuwenden, der auf die Techniken und Verfahren verweist, »die den Zweck haben, das Verhalten der Menschen zu steuern. Regierung der Kinder, Regierung der Seelen oder des Gewissens, Regierung eines Hauses, eines Staats oder von sich selbst« (Foucault [1980] 2005: 154). Dies impliziert im Weiteren ein solches Verständnis des Politischen, das die politische Verfassung der Gesellschaften, ihre Strukturen, Organisationsprinzipen, Institutionen, Gewaltformen und Rationalitäten als eine Verfestigung gesellschaftlicher Machtbeziehungen, als Ergebnis »eines Regimes vielfältiger Gouvernementalität « (Foucault [1984] 2000: 70) entziffert. Demnach müssen gerade diese Regierungstechnologien untersucht werden, denn sie erlauben es, gesellschaftliche Macht- und Herrschaftsverhältnisse als staatliche Politik festzuschreiben. 


\subsection{Rezeption des Konzepts}

Foucaults Untersuchungen zur Gouvernementalität und zur Problematisierung des modernen Staates wurden zuerst von seinen Kollegen und Zuhörern aufgegriffen (wie etwa von Roland Barthes, Daniel Defert, Gilles Deleuze, François Ewald, Alessandro Fontana, Jacques Lagrange, Pasquale Pasquino u.a.). Mit den ersten Übersetzungen seiner Werke in die englische Sprache, Ende 1980er Jahre, wurden seine Gedanken stärker im anglosächsischen Raum rezipiert (so etwa von Andrew Barry, Graham Burchell, Mitchell Dean, Colin Gordon, Timothy Luke, Paul Rabinow, Niklas Rose u.a.). Ende 1990er Jahre wuchs auch in dem deutschsprachigen Raum das Interesse an seinen analytischen Methoden; darunter auch an dem Konzept der Gouvernementalität (etwa bei Michael Bischoff, Ulrich Bröckling, Reiner Keller, Susanne Krasmann, Thomas Lemke, Martin Saar, Axel Honneth u.a.). Im Milleniumsjahr erschien dann die erste Studie zur Gouvernementalität der Gegenwart (vgl. Bröckling/Krasmann/Lemke 2000).

Die Heterogenität des Konzepts hat sich auch in seinen diversen Anwendungen widergespiegelt. Das Spektrum der Gouvernementalitäts-Studien breitet sich zum heutigen Tag weit aus über die gesamten Disziplinen der Geisteswissenschaften: von Geschichte, Philosophie, Soziologie, Pädagogik, Politikwissenschaft, Psychologie, Humangeografie, Anthropologie, Ökologie bis hin zu Gender-Studien, Kulturwissenschaft oder Theologie.

Inspiriert von der Theorie des Regierens, wie sie im Konzept der Gouvernementalität enthalten ist, fokussiert auch die Erforschung des Präventionsdispositivs darauf, seine Funktionsweise, seine Machteffekte und sein Bearbeiten individueller und gesellschaftlicher Probleme, wie die des Burnout-Syndroms, zu untersuchen und seine Einschreibung in das Regime der Gouvernementalität moderner Staaten zu verfolgen. 